\title{
ON SUCCESSIVE APPROXIMATIONS FOR NONEXPANSIVE MAPPINGS IN BANACH SPACES $\dagger$
}

\author{
by W. A. KIRK
}

(Received 16 September, 1969; revised 26 December, 1969)

Let $X$ be a Banach space and $K$ a convex subset of $X$. A mapping $T$ of $K$ into $K$ is called a nonexpansive mapping if $\|T(x)-T(y)\| \leqq\|x-y\|$ for all $x, y \in K$.

In general, it is not the case for nonexpansive mappings $T$ that the sequences of Picard iterates $\left\{T^{n}(x)\right\}$ converge to fixed points of $T$, and thus when such fixed points exist other approximation techniques are needed. One such technique is to form the mapping

$$
S_{\lambda}=\lambda I+(1-\lambda) T \quad(0<\lambda<1),
$$

and then show that under certain circumstances the Picard iterates of $S_{\lambda}$ converge to a fixed point of $T$. The first such result was obtained by Krasnoselskii [7], who proved that if $K$ is a closed convex subset of a uniformly convex Banach space and if $T$ is a nonexpansive mapping of $K$ into a compact subset of $K$, then for any $x \in K$ the sequence of iterates $\left\{S_{\lambda}^{n}(x)\right\}$, for $\lambda=1 / 2$, converges to a fixed point of $T$. It was noted by Schaefer [8] that this theorem holds for arbitrary $\lambda \in(0,1)$ and subsequently Edelstein [4] proved the corresponding result in a strictly convex Banach space. Even more recently, Browder and Petryshyn have obtained Krasnoselskii's theorem as a corollary of their results in [3].

Our purpose in this note is to observe that mappings more general than those of type $S_{\lambda}$ yield similar convergence theorems.

THEOREM 1. Let $K$ be a convex subset of a Banach space and $T$ a nonexpansive mapping of $K$ into itself. Define the mapping $S: K \rightarrow K$ by

$$
S=\alpha_{0} I+\alpha_{1} T+\alpha_{2} T^{2}+\ldots+\alpha_{k} T^{k}
$$

where $\alpha_{i} \geqq 0, \alpha_{1}>0$, and $\sum_{i=0}^{k} \alpha_{i}=1$. Then $S(x)=x$ if and only if $T(x)=x$.

Proof. Suppose $S(x)=x$. Then

$$
x=\sum_{i=1}^{k} \beta_{i} T^{i}(x),
$$

where $\beta_{i}=\alpha_{i} /\left(1-\alpha_{0}\right)$. Thus $x \in \operatorname{conv}\left\{T(x), T^{2}(x), \ldots, T^{k}(x)\right\}$. Let

$$
\delta=\sup \left\{\|u-v\|: u, v \in\left\{x, T(x), T^{2}(x), \ldots, T^{k}(x)\right\}\right\} .
$$

Because $T$ is nonexpansive, for some integer $p \geqq 1$,

$$
\left\|x-T^{p}(x)\right\|=\delta .
$$

† Research supported by the National Science Foundation, grant GP8367. 
Assume $\delta>0$, and let $p$ be the smallest positive integer such that $\left(^{*}\right)$ holds. Since $\alpha_{1}>0$,

$$
x=\beta_{1} T(x)+\left(1-\beta_{1}\right) z,
$$

where $z \in \operatorname{conv}\left\{T^{2}(x), T^{3}(x), \ldots, T^{k}(x)\right\}$ and $0<\beta_{1} \leqq 1$; thus

$$
\begin{aligned}
\delta=\left\|x-T^{p}(x)\right\| & =\left\|\beta_{1} T(x)+\left(1-\beta_{1}\right) z-T^{p}(x)\right\| \\
& \leqq \beta_{1}\left\|T(x)-T^{p}(x)\right\|+\left(1-\beta_{1}\right)\left\|z-T^{p}(x)\right\| \\
& \leqq \beta_{1} \delta+\left(1-\beta_{1}\right) \delta=\delta .
\end{aligned}
$$

This implies $\left\|T(x)-T^{p}(x)\right\|=\delta$, yielding $\left\|x-T^{p-1}(x)\right\| \geqq \delta$. This gives a contradiction if $p>1$. However, if $p=1$ the preceding argument yields $\|T(x)-T(x)\| \geqq \delta>0$, which is absurd. Thus, $\delta=0$ and $x=T(x)$. Since the converse is obvious, the theorem is proved. (We should remark that the stipulation $\alpha_{1}>0$ in Theorem 1 is necessary to rule out the possibility that a fixed point of $S$ is merely a point at which $T$ is periodic.) that is,

Next we prove that in uniformly convex spaces the mapping $S$ is asymptotically regular;

$$
\lim _{n \rightarrow \infty}\left\|S^{n+1}(x)-S^{n}(x)\right\|=0 \quad(x \in K) .
$$

This result is patterned after Theorem 5 in Browder and Petryshyn [3].

THEOREM 2. Let $X$ be uniformly convex and let $T$ and $S$ be as defined in Theorem 1. If $T$ has at least one fixed point then the mapping $S$ is asymptotically regular.

Proof. Let $x \in K$. Define the sequence $\left\{x_{n}\right\}$ by $x_{n}=S^{n} x, n=1,2, \ldots$ Suppose $u$ is a fixed point of $T$ in $K$. Then the sequence $\left\{\left\|x_{n}-u\right\|\right\}$ is nonincreasing (since $S$ is nonexpansive and $S(u)=u$ ), and we may suppose $\lim \left\|x_{n}-u\right\|=d \geqq 0$. Assume $d>0$. (If $d=0$ there is clearly nothing to prove.) Then (adopting the notation $T^{0}=I$ ) we have

$$
\begin{aligned}
x_{n+1}-u & =S\left(x_{n}\right)-u \\
& =\sum_{i=0}^{k} \alpha_{i} T^{\prime}\left(x_{n}\right)-u \\
& =\alpha_{0}\left(x_{n}-u\right)+\left(1-\alpha_{0}\right) z_{n},
\end{aligned}
$$

where

$$
z_{n}=\frac{1}{1-\alpha_{0}} \sum_{i=1}^{k} \alpha_{i}\left(T^{i}\left(x_{n}\right)-u\right)
$$

Since

\[ \left\|T^{i}\left(x_{n}\right)-u\right\|=\left\|T^{i}\left(x_{n}\right)-T^{i}(u)\right\| \leqq\left\|x_{n}-u\right\| \]
and $\sum_{i=0}^{k} \alpha_{i}=1$ it follows that $\limsup _{n \rightarrow \infty}\left\|z_{n}\right\| \leqq d$. Also $\lim _{n \rightarrow \infty}\left\|x_{n}-u\right\|=d, \lim _{n \rightarrow \infty}\left\|x_{n+1}-u\right\|=d$. 
Because $X$ is uniformly convex it must be the case that

$$
\lim _{n \rightarrow \infty}\left\|x_{n}-u-z_{n}\right\|=0 \text {. }
$$

However, $x_{n+1}-x_{n}=\left(1-\alpha_{0}\right)\left(x_{n}-u-z_{n}\right)$ and so $\lim \left(x_{n+1}-x_{n}\right)=0$, completing the proof.

The above results and Theorem 6 of [3] yield the following corollary.

Corollary. Let $X$ be a uniformly convex Banach space and $T$ a nonexpansive compact mapping of $X$ into $X$ (i.e., $T$ maps bounded subsets of $X$ into precompact subsets of $X$ ) which has at least one fixed point. Then if the mapping $S$ is defined as in Theorem 1 , for each $x_{0} \in X$ the sequence $\left\{S^{n}\left(x_{0}\right)\right\}$ converges to a fixed point of $T$.

Proof. Since $S$ is asymptotically regular and has the same fixed points as $T$, the conclusion is a direct consequence of Theorem 6 of Browder-Petryshyn [3] if it is the case that $I-S$ maps bounded closed subsets of $X$ into closed subsets of $X$. Let $H$ be a bounded closed subset of $X$ and suppose $\lim \left(h_{n}-S h_{n}\right)=z, h_{n} \in H$. We need to show that $z \in(I-S)[H]$. Since $T$ is a compact mapping, some subsequence $\left\{T\left(h_{n_{j}}\right)\right\}$ of $\left\{T\left(h_{n}\right)\right\}$ converges; say $T\left(h_{n j}\right) \rightarrow v$ as $j \rightarrow \infty$. Fix $i$ between 1 and $k$. Continuity of $T$ implies $T^{i}\left(h_{n_{j}}\right) \rightarrow T^{i-1}(v)$ as $j \rightarrow \infty$. Thus by repeatedly choosing subsequences, we may obtain a subsequence $\left(h_{n}\right\}$ of $\left\{h_{n}\right\}$ which has the property:

Now

$$
\lim _{n \rightarrow \infty} T^{i}\left(h_{n}\right)=w_{i} \in X \quad(i=1, \ldots, k)
$$

Since $\bar{h}_{n}-S\left(h_{n}\right) \rightarrow z$ as $n \rightarrow \infty$ it follows that

$$
\begin{aligned}
(I-S)\left(h_{n}\right) & =h_{n}-\sum_{i=0}^{k} \alpha_{i} T^{i}\left(h_{n}\right) \\
& =\left(1-\alpha_{0}\right) h_{n}-\sum_{i=1}^{k} \alpha_{i} T^{i}\left(h_{n}\right) .
\end{aligned}
$$

$$
\lim _{n \rightarrow \infty}\left(1-\alpha_{0}\right) h_{n}=z+\sum_{i=1}^{k} \alpha_{i} w_{i} .
$$

This implies that $\left\{h_{n}\right\}$ converges, say to $h \in H$ (since $H$ is closed). Hence $h-S h=z$, which completes the proof.

We conclude by giving an analogue of Theorem 7 of Browder [2].

THEOREM 3. Let $X$ be a uniformly convex Banach space, $K$ a closed bounded convex subset of $X$, and $T$ a nonexpansive mapping of $K$ into $K$. Let

$$
S=\sum_{i=0}^{k} \alpha_{i} T^{i}
$$

where $\alpha_{i} \geqq 0, \alpha_{1}>0$, and $\sum_{i=0}^{k} \alpha_{i}=1$. Suppose $T$ has at most one fixed point $y$ in $K$. Then for each $x_{0}$ in $K$ the sequence $\left\{S^{n}\left(x_{0}\right)\right\}$ converges weakly to $y$ in $K$.

Proof. Since $S$ is nonexpansive, Theorem 3 of [2] implies that $I-S$ is demiclosed. This means that if $\left\{u_{j}\right\}$ converges weakly to $u_{0}$ in $K$ and $(I-S)\left(u_{j}\right)$ converges strongly to $w$, then $(I-S)\left(u_{0}\right)=w$. 
Now let $x_{n}=S^{n}\left(x_{0}\right), n=1,2, \ldots$, and suppose $\left\{x_{n_{i}}\right\}$ converges weakly to $u_{0}$. By Theorem $2, S$ is asymptotically regular so

$$
\lim _{i \rightarrow \infty}(I-S)\left(x_{n_{i}}\right)=\lim _{i \rightarrow \infty}\left(S^{n_{1}}\left(x_{0}\right)-S^{n_{i}+1}\left(x_{0}\right)\right)=0
$$

and thus demiclosedness of $I-S$ implies

$$
(I-S)\left(u_{0}\right)=0 \text {. }
$$

Thus $u_{0}$ is a fixed point of $S$. However, by Theorem 1 the fixed points of $S$ and $T$ coincide. Therefore $u_{0}$ is the unique fixed point of $T$ and it follows that every weakly convergent subsequence of $\left\{x_{n}\right\}$ converges weakly to $u_{0}$. If $\left\{x_{n}\right\}$ does not converge weakly to $u_{0}$ then there exists a weak neighborhood $W$ of $u_{0}$ and a subsequence $\left\{x_{n_{k}}\right\}$ of $\left\{x_{n}\right\}$ with the property that $x_{n_{k}} \notin W, k=1,2, \ldots$ However, reflexivity of $X$ and boundedness of $\left\{x_{n}\right\}$ imply that some subsequence of $\left\{x_{n_{k}}\right\}$ converges weakly, and by what we have just shown, this weakly convergent subsequence must converge to $u_{0}$. This implies that terms of the sequence $\left\{x_{n_{k}}\right\}$ must lie in $W$-a contradiction. Therefore, $\left\{S^{n}\left(x_{0}\right)\right\}$ converges weakly to $u_{0}$.

We might remark that the existence of at least one fixed point for $T$ in $K$ follows from a theorem proved independently by Browder [1], Göhde [5], and Kirk [6]. In general, this fixed point is not unique, but it will be unique for strictly contractive mappings (i.e., mappings $T$ for which $\|T(x)-T(y)\|<\|x-y\|$ when $x \neq y$ ).

Added IN Proof. Using Theorem 1, one may also obtain Theorems 2 and 3 as direct consequences of their analogues in [2] and [3] by applying the original theorems to the mapping

$$
R=\left(\frac{1}{1-\alpha_{0}}\right) \sum_{i=1}^{k} \alpha_{i} T^{i}
$$

\section{REFERENCES}

1. F. E. Browder, Nonexpansive nonlinear operators in a Banach space, Proc. Nat. Acad. Sci. U.S.A. 54 (1965), 1041-1044.

2. F. E. Browder, Semicontractive and semiaccretive nonlinear mappings in Banach spaces, Bull. Amer. Math. Soc. 74 (1968), 660-665.

3. F. E. Browder and W. V. Petryshyn, The solution by iteration of nonlinear functional equations in Banach spaces, Bull. Amer. Math. Soc. 72 (1966), 571-575. 509-510.

4. M. Edelstein, A remark on a theorem of M. A. Krasnoselskii, Amer. Math. Monthly 73 (1966),

5. D. Göhde, Zum Prinzip der kontraktiven Abbildung, Math. Nachr. 30 (1965), 251-258.

6. W. A. Kirk, A fixed point theorem for mappings which do not increase distances, Amer. Math. Monthly 72 (1965), 1004-1006.

7. M. A. Krasnoselskii, Two remarks about the method of successive approximations, Uspehi Mat. Nauk 10 (1955), no. 1 (63), 123-127.

8. H. Schaefer, Uber die Methode suksessiver Approximation, Jber. Deutsch. Math. Verein. 59 (1957), 131-140.

UNIVERSITY OF IOWA

IOWA CITY

IOWA 52240, U.S.A. 